\title{
A Versatile Multiple Time Step Scheme for Efficient $a b$ Initio Molecular Dynamics Simulations
}

\author{
Elisa Liberatore, Rocco Meli, ${ }^{(i)}$ and Ursula Rothlisberger**i) \\ École Polytechnique Fédérale de Lausanne (EPFL), CH-1015 Lausanne, Switzerland
}

Supporting Information

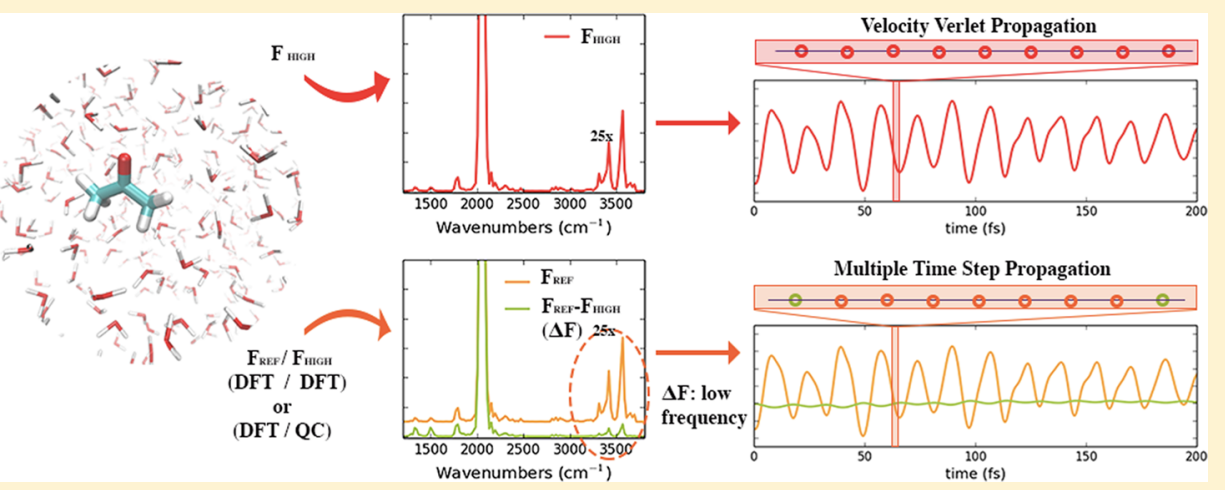

ABSTRACT: We present here our implementation of a time-reversible, multiple time step (MTS) method for full QM and hybrid QM/MM Born-Oppenheimer molecular dynamics simulations. The method relies on a fully flexible combination of electronic structure methods, from density functional theory to wave function-based quantum chemistry methods, to evaluate the nuclear forces in the reference and in the correction steps. The possibility of combining different electronic structure methods is based on the observation that exchange and correlation terms only contribute to low frequency modes of nuclear forces. We show how a pair of low/high level electronic structure methods that individually would lead to very different system properties can be efficiently combined in the reference and correction steps of this MTS scheme. The current MTS implementation makes it possible to perform highly accurate $a b$ initio molecular dynamics simulations at reduced computational cost. Stable and accurate trajectories were obtained with time steps of several femtoseconds, similar to and even exceeding the ones usually adopted in classical molecular dynamics, in particular when using a generalized Langevin stochastic thermostat. Compared to the standard Velocity Verlet integration, the present MTS scheme allows for a 5- to 6-fold overall speedup, at an unaltered level of accuracy.

\section{INTRODUCTION}

$A b$ initio molecular dynamics (AIMD) simulations are valuable tools to explore static, dynamical, and electronic properties of biomolecular and condensed phase systems at a high level of accuracy, without relying on preconstructed, empirical force fields. On the other hand, in standard Born-Oppenheimer AIMD the evaluation of the forces acting on the nuclei requires a calculation of the electronic ground (or excited) state energy and its derivatives with respect to the nuclear positions at each MD step: As ab initio force calculations are orders of magnitude more computationally expensive than force evaluations based on empirical force fields, AIMD simulations are only feasible for small size systems (few hundreds of atoms) and short time windows (hundreds of picoseconds). For a fixed-size problem the computational cost of an $\mathrm{MD}$ simulation depends on two competing time scales: the time scale of the relevant phenomena determined by the properties one wants to measure (conformational changes, reaction mechanisms, ...) that defines the total simulation time and the time scale of the fastest internal motions, $T_{\text {fast }}$, that poses an upper bound to the time step $\delta t$ used to integrate the equations of motion. In the Velocity Verlet (VV) algorithm, ${ }^{1}$ a standard integrator in classical and $a b$ initio $\mathrm{MD}, \delta t$ must satisfy $\delta t<T_{\text {fast }} / \pi$ to achieve stable dynamics. ${ }^{2}$ However, much smaller values of $\delta t$, of the order of a tenth of a femtosecond, are generally recommended to avoid large energy fluctuations or artifacts such as spectral shifts of the fastest vibrational frequencies.

A possible route to increase the efficiency of AIMD simulations consists of designing algorithms which support larger integration time steps. Constrained algorithms were introduced to freeze the fastest degrees of freedom (DoF), hence removing the high-frequency modes from the system. While this approach allows for an increase in the time step, it might determine an alteration of the system properties. ${ }^{5,6}$ It also requires a careful selection of the degrees of freedom to be constrained based on an a priori knowledge of those contributing to the high-frequency region of the vibrational

Received: November 27, 2017

Published: April 6, 2018 
spectrum, not always easy-to-identify in large molecular systems. An alternative and more general approach to enable larger time steps in $\mathrm{MD}$ is provided by multiple time step (MTS) methods.

Multiple time step algorithms, ${ }^{7-16}$ originally derived for classical $\mathrm{MD}$, exploit the time scale separation between faster DoF, which can be integrated more frequently, and slower DoF, which can be updated at a comparably lower rate. This approach can lead to a considerable gain in terms of computational time when the slow forces are also the most expensive. In a series of seminal papers published in the early 1990s, ${ }^{7-11,17}$ Tuckerman and co-workers presented several MTS algorithms for systems with classical forces (stiff and soft forces, long-range and short-range forces, disparate masses) which have also the quality of being time-reversible. Their microcanonical, reversible reference system propagation algorithm (rRESPA) ${ }^{11}$ has lately been used as a model for a multiplicity of MTS variants applied to $a b$ initio MD, in which a time scale separation is artificially introduced via suitable splittings of the electronic Hamiltonian, ${ }^{18}$ combining basis sets of different size $\mathrm{e}^{19}$ or forces calculated $a b$ initio at varying accuracy. ${ }^{20-22}$ In the scheme proposed by Steele, ${ }^{20}$ HartreeFock (HF) forces have been used in the reference steps and second-order Møller-Plesset perturbation theory $(\mathrm{MP} 2)^{23}$ contributions were added in the correction steps. As an alternative, in ref 21, reference and high level forces have been calculated at the same level of theory, HF or density functional theory (DFT) with hybrid exchange and correlation (XC) functionals, but varying the two-electron integral screening. A similar approach has been used by Guidon et al. within DFT, combining forces from generalized gradient approximation and hybrid XC functionals. ${ }^{22}$ rRESPA has been later extended to thermostated versions which attenuate or even eliminate the resonances emerging when the time step approaches the $T_{\text {fast }} / \pi$ limit and thus allow for even larger time steps in classical $\mathrm{MD}^{24-26}$

In this paper, we present our MTS scheme for accelerating Born-Oppenheimer $a b$ initio MD (BOMD), derived as an adaptation of the rRESPA algorithm (see section 2). ${ }^{11}$ The algorithm has been implemented in the DFT-based, plane-wave code $\mathrm{CPMD}^{27}$ and includes a customizable interface with quantum chemistry (QC) codes, allowing for a flexible combination, in the reference and in the correction steps, of different couples of electronic state methods. For instance, DFT XC functionals of different accuracy-from Local Density Approximation (LDA) ${ }^{28}$ to hybrids and meta-hybrids - can be mixed or the dynamics can be corrected with post-HF methods such as MP2.

The assessment of the performances of the present scheme in terms of accuracy and efficiency has been carried out on simple test systems on the basis of qualitative and quantitative comparison of static and dynamic properties, as described in sections 3 and 4. The algorithm allows for a 10-fold increase in the time step and, thanks to a combination with a colored noise thermostat based on Generalized Langevin Equations (GLE), ${ }^{26,29,30}$ up to a 6-fold speedup. A summary of applicability, advantages, and drawbacks of this MTS implementation is presented in the conclusive section 5 .

\section{THEORY}

Before introducing our implementation in detail, we recall here the main steps of the rRESPA algorithm ${ }^{11}$ derivation, useful to establish the general notation.
In the Lagrange formalism, the time evolution from an initial time $t$ to a time $t^{\prime}$ of a phase space element $\Gamma(\mathbf{q}, \mathbf{p} ; t)$ can be obtained via the application of a time propagator $G\left(t^{\prime}, t\right)$ as in

$$
\begin{aligned}
\Gamma\left(\mathbf{q}, \mathbf{p} ; t^{\prime}\right) & =G_{\mathbf{q}, \mathbf{p}}\left(t^{\prime}, t\right) \Gamma(\mathbf{q}, \mathbf{p} ; t) \\
& =e^{i \mathcal{L}_{\mathbf{q}, \mathbf{p}}\left(t-t^{\prime}\right)} \Gamma(\mathbf{q}, \mathbf{p} ; t)
\end{aligned}
$$

where $\mathbf{q}$ and $\mathbf{p}$ are the canonically conjugated, $3 \mathrm{~N}$ dimensional coordinates (positions and momenta, respectively) and $\mathcal{L}$ is the Liouville operator, that, highlighting the "slow" and "fast" contributions of the nuclear forces and assuming for simplicity that all the particles in the system have the same mass $m$, can be split up into

$$
\begin{aligned}
i \mathcal{L}_{\mathbf{q}, \mathbf{p}} & =i \mathcal{L}_{\mathbf{q}}^{\text {slow }}+i \mathcal{L}_{\mathbf{q}}^{\text {fast }}+i \mathcal{L}_{\mathbf{p}} \\
& =\mathbf{F}^{\text {slow }}(\mathbf{q}) \frac{\partial}{\partial \mathbf{p}}+\mathbf{F}^{\text {fast }}(\mathbf{q}) \frac{\partial}{\partial \mathbf{p}}+\frac{\mathbf{p}}{m} \frac{\partial}{\partial \mathbf{q}}
\end{aligned}
$$

Notice that in the above we adopted for brevity the Einstein notation, omitting the sum over the particle indices. Within this formulation, the Velocity Verlet algorithm is obtained by separating, via symmetric Trotter factorization, the kinetic and the potential part of the classical propagator, $\exp \left(i \mathcal{L}_{\mathbf{q}} t\right)$ and $\exp \left(i \mathcal{L}_{\mathbf{p}} t\right)$. The idea underlying rRESPA is to split the contributions of the forces on the nuclear degrees of freedom according to the time scale of their variation by a suitable, symmetrical Trotter factorization of the classical Liouville propagator. The classical propagator can then be written as the product:

$$
G(\Delta t) \approx e^{i \mathcal{L}_{\mathbf{q}}^{\text {slow }} \Delta t / 2} e^{i\left(\mathcal{L}_{\mathbf{q}}^{\text {fast }}+\mathcal{L}_{\mathbf{p}}\right) \Delta t} e^{i \mathcal{L}_{\mathbf{q}}^{\text {slow }} \Delta t / 2}
$$

Adjusting the time step $\Delta t$ to the slower time scale requires an additional factorization of the middle term, which leads to the final expression of the MTS propagator:

$$
G(\Delta t) \approx e^{i \mathcal{L}_{\mathbf{q}}^{\text {slow }} \Delta t / 2}\left[e^{i\left(\mathcal{L}_{\mathbf{q}}^{\text {fast }}+\mathcal{L}_{\mathbf{p}}\right) \Delta t / n}\right]^{n} e^{i \mathcal{L}_{\mathbf{q}}^{\text {slow }} \Delta t / 2}
$$

The action of the inner term is equivalent to $n$ Velocity Verlet propagations ${ }^{1}$ with the fast forces only and with a time step $\delta t=$ $\Delta t / n$. The whole MTS propagation can then also be seen as a standard VV propagation in which the total forces have an impulsive component: $\mathbf{F}=\mathbf{F}^{\text {fast }}+n \mathbf{F}^{\text {slow }} \delta(t-n \Delta t)$.

2.1. Multiple Time Step Implementation within $a b$ Initio MD. The key property that makes it possible to apply a multiple time step algorithm such as rRESPA is the existence of two well separated time scales in the system. These time scales can have a physical origin (as in the case of "slow" long-range forces and "fast" short-range forces in classical MD), but can also be introduced artificially, re-expressing ad hoc the interactions in the systems to partition them into two contributions: a "fast" component of the reference forces and a "slow" component corresponding to the correction term.

In our algorithm, the propagator is formally identical to rRESPA, with the difference that the forces are calculated $a b$ initio instead of being derived from an analytic potential. To climb Jakob's ladder of the exchange and correlation functionals, a functional on a lower rung is used as a reference while a functional on a higher rung is used only at the larger time step to evaluate the correction.

The forces from the lower functional, $\mathbf{F}^{\mathrm{L}}$, play the role of the "fast" forces, while the "slow" forces are given by the difference $\mathbf{F}^{\text {slow }}=\mathbf{F}^{\mathrm{H}}-\mathbf{F}^{\mathrm{L}}=\Delta \mathbf{F}$, where $\mathbf{F}^{\mathrm{H}}$ are the forces calculated at a 

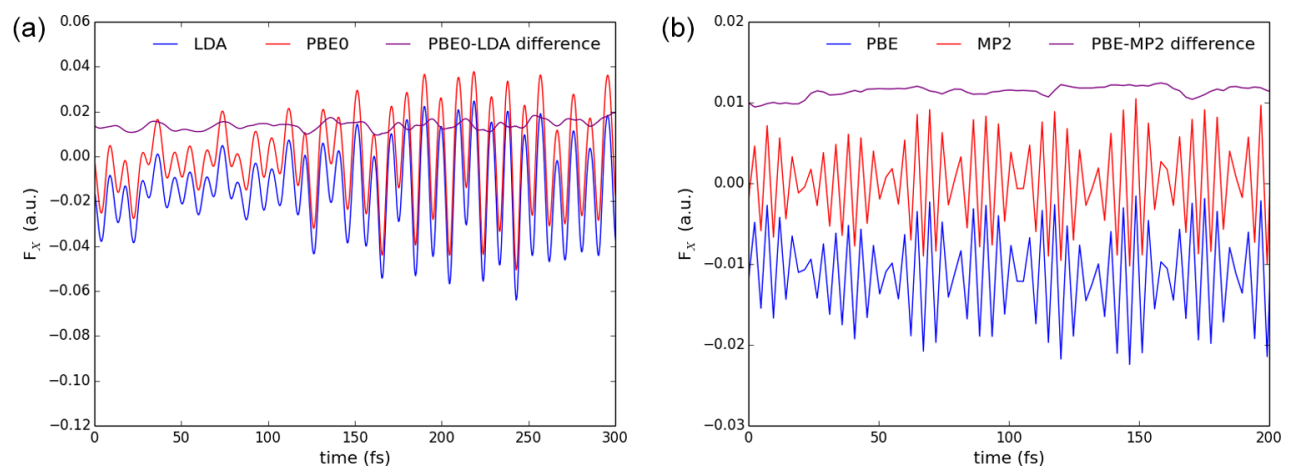

Figure 1. $x$-Component of the total force and of the force difference $F$, along a portion of the MTS trajectory on (a) an oxygen atom in a system of 32 water molecules, calculated using LDA and PBE0 and (b) the hydrogen bond acceptor in a water dimer, calculated using PBE and MP2.

higher level of accuracy. As exchange and correlation terms only give a minor contribution to the total energy and have no explicit dependence on nuclear coordinates, it is reasonable to assume that the difference $\Delta \mathrm{F}$ will be small and smoothly vary with time. Our observation finds its empirical confirmation in Figure 1a, which illustrates the time evolution along an MTS trajectory of the $x$-component of the forces $\mathbf{F}^{\mathrm{H}}$ and $\mathbf{F}^{\mathrm{L}}$ on an oxygen atom in liquid water obtained using the $\mathrm{PBEO}^{31}$ and the LDA functional, respectively. Comparison of the "pure" forces with their difference $\Delta \mathbf{F}$ clearly highlights the different time scales of their variation, on which the applicability of the MTS scheme relies. Similarly, Figure $1 \mathrm{~b}$ compares the $x$-component of forces on the $\mathrm{H}$-bonded oxygen in a water dimer evaluated at the DFT-PBE ${ }^{32}$ and at the MP2 level along the same MD trajectory. Also in this case the difference between the forces is small and slowly varies with time compared to each of the "pure" forces. This suggests that the MTS propagation can be applied also using DFT in the reference steps and a different electronic structure method in the correction steps.

We implemented our MTS scheme in a development version of the DFT-based code for ab initio molecular dynamics CPMD v 4.1. ${ }^{27}$ For a combination of DFT and wave function-based quantum chemistry methods, we implemented an interface with the quantum chemistry codes GAUSSIAN09 (G09) ${ }^{33}$ and TURBOMOLE. $^{34}$ This interface makes it possible to calculate $\mathbf{F}^{\mathrm{H}}$ using post-Hartree-Fock methods such as configuration interaction (CI) or order- $n$ Møller-Plesset perturbation theory $(\mathrm{MPn})$ and can be easily customized to select any of the methods provided by G09, TURBOMOLE, or other packages.

The MTS scheme presented here can be used in full QM as well as in hybrid QM/MM calculations, for microcanonical and for canonical sampling. In the latter case, any of the thermostats available in CPMD can be chosen. The canonical propagator can be derived in the Lagrange formalism. If for instance a Nosé-Hoover thermostat ${ }^{55-37}$ is applied, the total Liouville operator will include additional terms relative to the coordinates and conjugate momenta of the thermostat, $\boldsymbol{\eta}$ and $\mathbf{p}_{\eta}$, and its interaction with the system.

$$
\begin{aligned}
i \mathcal{L} & =i \mathcal{L}_{\mathbf{q}, \mathbf{p}}+i \mathcal{L}_{\boldsymbol{\eta}, \mathbf{p}_{\eta}, \mathbf{p}}^{\mathrm{NH}} \\
& =i \mathcal{L}_{\mathbf{q}}^{\text {slow }}+i \mathcal{L}_{\mathbf{q}}^{\text {fast }}+i \mathcal{L}_{\mathbf{p}} \\
& +i \mathcal{L}_{\mathbf{p}_{\eta}}+i \mathcal{L}_{\boldsymbol{\eta} \mathbf{p}}+i \mathcal{L}_{\boldsymbol{\eta}}
\end{aligned}
$$

Given this form of $i \mathcal{L}$, there are two possible, equally valid strategies of MTS factorization: the thermostat variables can be considered part of the "fast" terms, as in ref 11 , or of the "slow" term. We opted for the second choice which corresponds to thermostatting only the physically meaningful velocities, those of the outer steps, and allows for good temperature control. Our NVT-MTS propagator is hence ${ }^{38}$

$$
\begin{aligned}
G(\Delta t) & \approx e^{i \mathcal{L}^{\mathrm{NH}} \Delta t / 2} e^{i \mathcal{L}_{\mathbf{q}}^{\text {slow }} \Delta t / 2} \\
& \times\left[e^{i\left(\mathcal{L}_{\mathbf{q}}^{\text {fast }}+\mathcal{L}_{\mathbf{p}}\right) \Delta t / n}\right]^{n} e^{i \mathcal{L}_{\mathbf{q}}^{\text {slow }} \Delta t / 2} e^{i \mathcal{L}^{\mathrm{NH}} \Delta t / 2}
\end{aligned}
$$

Alternatively, if the thermostat variables are included in the "fast" term different options are possibile. In particular, the socalled middle-scheme factorization of the canonical propagator,

$$
G(\delta t)=e^{i \mathcal{L}_{\mathrm{p}} \delta t / 2} e^{i \mathcal{L}_{\mathrm{q}} \delta t / 2} e^{i \mathcal{L}^{\mathrm{NH}} \delta t} e^{i \mathcal{L}_{\mathrm{q}} \delta t / 2} e^{i \mathcal{L}_{\mathrm{p}} \delta t / 2}
$$

in which the thermostat operator is in the center of the Trotter expansion, has been recently found ${ }^{39}$ to be more accurate than other schemes and can also be used as a basis of an NVT-MTS propagation (see Supporting Information).

Besides mere temperature control, the use of thermostats has also additional advantages in MTS dynamics. The major obstacle to MTS efficiency is the occurrence of resonances ${ }^{40}$ that are due to insufficient dynamical decoupling and prevent the use of larger time steps and time step ratios. For a harmonic oscillator, linear resonances arise when the outer time step equals or exceeds the time period of the fastest vibration, $T_{\text {fast }}$, divided by $\pi, \Delta t_{\max }=T_{\text {fast }} / \pi$, hence limiting the maximum value of the outer time step to a few femtoseconds for typical covalently bonded systems. The problem has been widely investigated, and at least three different approaches based on thermostats have been proposed in order to eliminate or reduce the resonance problem in classical MTS-MD and push the outer time step to larger values. In the "Iso-NHC-RESPA" by Minary et al. ${ }^{24}$ a Nosé-Hoover chain coupled to the physical momenta through isokinetic constraints is used to remove kinetic energy from the system, stabilizing the resonances. The "Stochastic-Iso-NH-RESPA" by Leimkuhler et $\mathrm{al}^{25}$ is conceptually similar, but to achieve higher computational efficiency and better performances it replaces the Nosé-Hoover chain with a stochastic Nosé-Hoover thermostat. Finally, the colored noise thermostat based on Generalized Langevin Equations (GLE) of Morrone et al., ${ }^{29,30}$ designed to enhance canonical sampling in path integral simulations or to mimic nuclear quantum effects in $\mathrm{MD}$ for classical nuclei, can be used to increase the outer time step in MTS dynamics. ${ }^{26}$ In the Discussion section we will show the performance improvement of our $a b$ initio MTS scheme when the GLE thermostat, already available in the CPMD code, is applied in the velocity correction steps. 

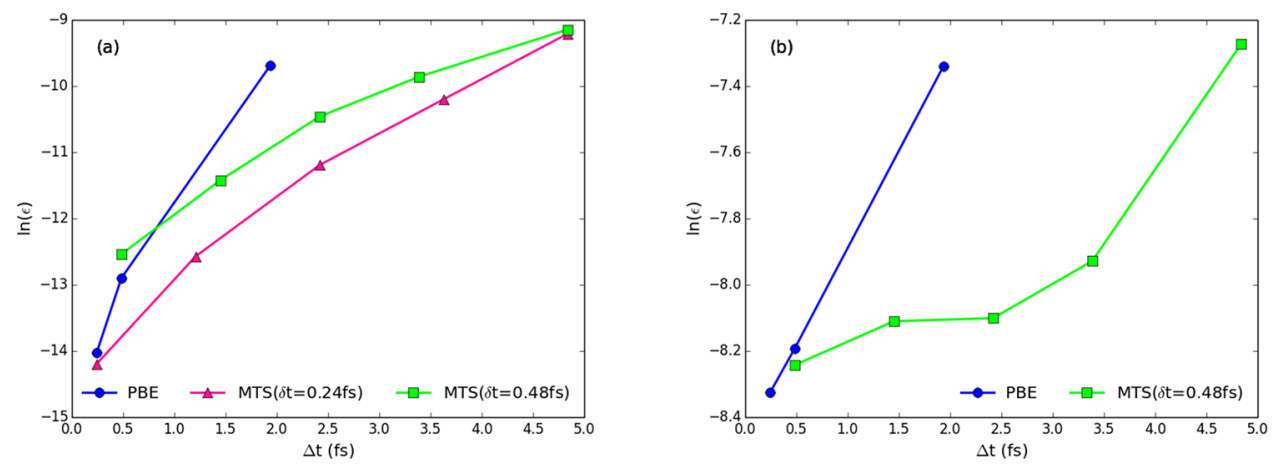

Figure 2. Logarithm of the average total energy fluctuation $\varepsilon$ as a function of the time step of integration, over VV and MTSn NVE trajectories of 26 ps for (a) an isolated acetone molecule and (b) an acetone molecule in water. All the trajectories relative to the same system were started from the same initial conditions, as described in section 3.1. Lines are visual guides.

\section{COMPUTATIONAL DETAILS}

We tested the implementation and the performances of the above-described MTS algorithm on three test systems. An acetone molecule in vacuum and in an aqueous solution of 3524 water molecules were used to test the general implementation of the full quantum and QM/MM MTS schemes, respectively. The interface with the external quantum chemistry codes G09 and TURBOMOLE was tested on an isolated water dimer. In small systems, such as a single acetone molecule, the computational cost of the high level calculation is not significantly higher than the one at a low level; hence, the computational gain of the MTS approach is marginal. To give a more realistic estimate of the speedup that can be reached through MTS propagation, we considered a periodically repeated system of 32 water molecules.

Before proceeding with a summary of the computational details of our simulations, it can be useful to clarify the nomenclature adopted throughout the paper to discriminate among the various types of simulations performed. The labels VV and MTS will be used to distinguish the standard Velocity Verlet integration from MTS propagation. These labels can be preceded by the ensemble indication as in NVE-VV or NVTMTS, and in the case of the MTS dynamics, the reference functional and the functional used in the correction can be indicated as REF/HIGH pairs (e.g., LDA/PBE0 NVE-MTS or PBE/MP2 NVT-MTS).

3.1. Acetone. In the full QM simulations of a single acetone molecule and in the $\mathrm{QM} / \mathrm{MM}$ simulations of an acetone molecule in water solution, the quantum forces were calculated at the DFT level using PBE (reference and correction steps) and PBE0 (correction steps) approximations for the XC functional. Wave functions and electron densities were expanded in a plane-wave basis set using an energy cutoff of 70 and $280 \mathrm{Ry}$, respectively. Core electrons were implicitly taken into account using norm-conserving Troullier-Martins ${ }^{41}$ pseudopotentials. The ab initio calculations for the QM region/ QM system were performed for a pseudoisolated system using the Martyna-Tuckerman Poisson solver. ${ }^{42}$ The QM simulation box was cubic and had a $10 \AA$ side. In the $\mathrm{QM} / \mathrm{MM}$ simulations, the acetone molecule was solvated in a $33.24 \AA \times$ $32.64 \AA \times 33.02 \AA$ box of TIP $3 \mathrm{P}^{43}$ water molecules.

For each system-isolated or solvated-we ran a total of seven NVE simulations: two reference Velocity Verlet runs, using the PBE functional and the PBE0 functional and the time step $\delta t=0.48 \mathrm{fs}$, and five MTS runs with the same value of the inner time step $(\delta t=0.48 \mathrm{fs})$ and different values of the outer time step, $\Delta t=0.48,1.44,2.40,3.36,4.80 \mathrm{fs}$, corresponding to $n=1,3,5,7$, and 10 times the inner time step.

For the isolated acetone molecule we performed an additional series of seven simulations: two reference VV runs and five MTSn runs, with a smaller value of $\delta t, \delta t=0.24 \mathrm{fs}$, in order to investigate the impact of the inner time step on the quality of the MTS dynamics. All the runs relative to the same physical system were started from the same initial conditions, obtained after an NVT equilibration of $5 \mathrm{ps}$, at $T=300 \mathrm{~K}$, that, in the case of the QM/MM system, followed a classical equilibration run. The total length of the NVE trajectories was $30 \mathrm{ps}$, of which only the last 26 ps were used for analysis.

3.2. Liquid Water. To obtain a more realistic assessment of the MTS speedup, we simulated a system of 32 water molecules, contained in a cubic simulation box of side $L=$ $9.939 \AA$ \&, with periodic boundary conditions. The density of the system corresponded to the experimental conditions for liquid water at atmospheric pressure and at temperature $T=350 \mathrm{~K}$, and it was chosen to match previous simulations of water with DFT hybrid functionals. ${ }^{44}$ The interactions with core electrons were accounted for by Trouiller-Martins pseudopotentials. The plane-wave cutoff for wave function and densities were 70 and $280 \mathrm{Ry}$, respectively. We performed a total of four simulations, all starting from the same initial geometry and velocities, taken from a pre-equilibrated PBE VV-NVT run at $350 \mathrm{~K}$ : (i) An LDA/PBE0 NVE-MTS simulation, with inner time step $\delta t=0.36 \mathrm{fs}$ and a four times longer outer time step, $\Delta t=1.44$ fs. (ii) An LDA/PBE0 NVT-MTS simulation, using the colored noise stochastic thermostat $\mathrm{GLE}^{26}$ with inner time step $\delta t=0.36 \mathrm{fs}$ and a 10 times longer outer time step, $\Delta t=$ 3.60 fs. (iii) An LDA NVE-VV and (iv) a PBEO NVE-VV simulation, both with time step $\delta t=0.36$ fs. The total length of the trajectories (i), (ii), and (iii) was 25 ps while the PBEO trajectory was shorter (13 ps); in all the cases, the initial $5 \mathrm{ps}$ were discarded from the analysis.

The values of the parameters determining the form of the memory kernel in the GLE equations correspond to those optimized and tested by the thermostat developers in ref 26: bath frequency $\omega=1600 \mathrm{~cm}^{-1}$, effective friction $\gamma_{\infty}=83.3 \mathrm{ps}^{-1}$, and white noise strength $\gamma_{0}=0.01 \mathrm{ps}^{-1}$.

3.3. Water Dimer. An isolated water dimer was chosen to test the interface between CPMD and G09 or TURBOMOLE. For the CPMD calculation, the PBE functional, TrouillerMartins pseudopotentials, an energy cutoff of $90 \mathrm{Ry}$, a cubic simulation box of $15 \AA$, and the Martyna-Tuckermann Poisson solver were adopted. The high level forces were obtained at the 

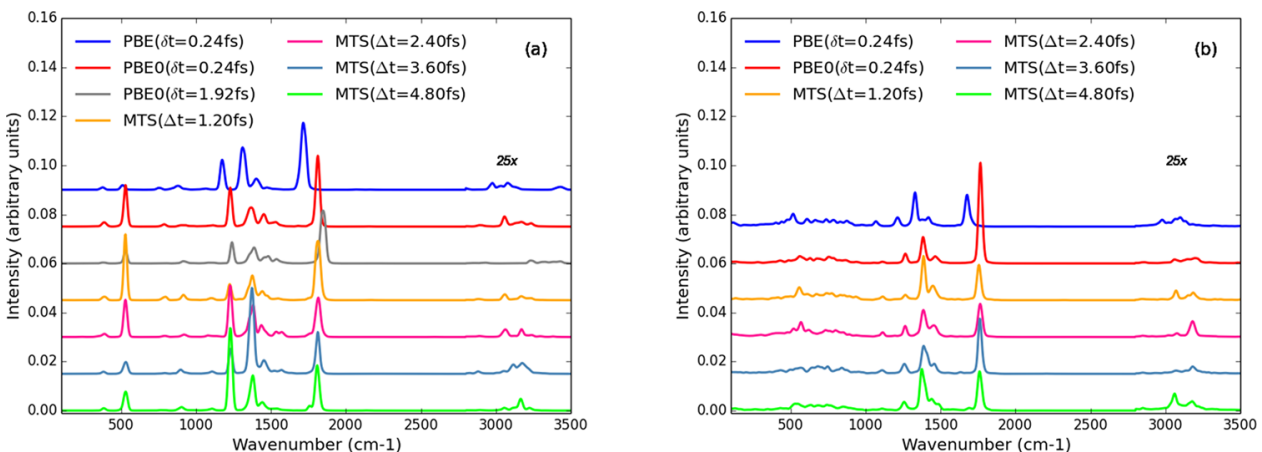

Figure 3. Vibrational spectra of acetone (a) in vacuum and (b) in solution, calculated along PBE/PBE0-MTSn trajectories of different outer time step and along VV PBE and PBE0 trajectories. For better visualization, a Gaussian smoothing has been applied to the raw spectra and the intensities of the bands around $3000 \mathrm{~cm}^{-1}$ have been magnified by a factor 25; the different curves are displayed with a different vertical shift.

MP2 level of theory from G09 and TURBOMOLE, using an aug-cc-pVTZ basis set. We ran two trajectories both with an inner step $\delta t=0.36 \mathrm{fs}$ and outer steps $\Delta t=1.08 \mathrm{fs}$ (MTS3) and $\Delta t=1.80 \mathrm{fs}$ (MTS5), of a total length equal to $10 \mathrm{ps}$. Given the relatively low strength of the hydrogen bond, to ensure the stability of the dimer, the simulations were performed in the NVT ensemble rather than NVE, applying the Nosé-Hoover thermostat at the outer time step only.

\section{DISCUSSION}

In a multiple time step dynamics the length of the outer time step $\Delta t$, fixing all the other computational parameters, determines both the efficiency of the calculation and the accuracy of the sampled trajectory. The choice of the ratio between the outer and inner time step is then the result of a compromise between efficiency and accuracy.

4.1. Quality Assessment. The quantity that is usually monitored, a posteriori, to evaluate the quality of the dynamics is the time evolution of a conserved quantity. In particular, the reliability of a microcanonical MD trajectory can be quantified by analyzing the size of the energy fluctuations:

$$
\varepsilon=\frac{1}{N} \sum_{i=1}^{N}\left|\frac{E_{i}-\bar{E}}{\bar{E}}\right|
$$

where $E_{i}$ and $\bar{E}$ are, respectively, the instantaneous and the average total energy calculated along a trajectory of $N$ steps. Figure 2 compares the total energy fluctuations obtained in $\mathrm{MTS} n$ trajectories with those for VV relative to the same system: acetone in vacuum (panel a) and in aqueous solution (panel b). The MTS curves always lie below the VV ones and have a smaller slope: in both systems the outer time step in the MTS trajectories can be enlarged up to $\Delta t \approx 2.5$ fs without significant accuracy loss. Stable trajectories, although with larger energy fluctuations, can even be obtained with $\Delta t \approx 4.0 \mathrm{fs}$. The effect of the inner time step on the stability can also be inferred by comparing, in Figure 2a, the MTS $n$ curves relative to $\delta t=$ 0.24 fs (pink triangles) and $\delta t=0.48$ fs (green squares). The offset between the curves is due to the different accuracy level of the VV-like integration of the inner time steps and it diminishes as the outer time step increases, meaning that, only for the smallest values of the outer time step, a more frequent VV integration in the intermediate steps helps reduce the total energy fluctuations.

A further assessment of the correctness of the sampling can be done by comparing structural (e.g., radial distribution function) and dynamical (e.g., anharmonic vibrational spectra) properties obtained from standard and MTS sampling, as illustrated in the following sections.

4.1.1. Dynamical Properties: Vibrational Frequencies of Acetone in Vacuum and in Solution. Vibrational spectra including anharmonic effects can be obtained as the Fourier transform of the dipole-dipole autocorrelation function calculated along the MD trajectory. The vibrational spectra of acetone in vacuum and in aqueous solution from VV and PBE/ PBE0-MTS $n$ trajectories are shown in Figure 3. The position and the broadening of the reference PBE0-VV spectral peaks are nicely reproduced in all the MTS-based spectra and are clearly blue-shifted compared to the PBE-VV spectra, meaning that the vibrational frequencies at the PBE0 level are correctly reproduced in the MTS dynamics, even at the largest $\Delta t=4.8$ fs. The MTS trajectories also show superior accuracy compared to VV trajectories of large time steps: in the acetone spectra computed along a PBE0-VV trajectory with the time step $\delta t=$ $1.92 \mathrm{fs}$ (see Figure 3a), the frequencies of the $\mathrm{CH}_{3}$ modes around $3000 \mathrm{~cm}^{-1}$ and of the CO stretch mode around 1800 $\mathrm{cm}^{-1}$ are shifted to higher wavenumbers with respect to the reference. The peak heights, related to the population of the vibrational levels, display a larger variation in the different trajectories. This disagreement can however be expected as the intensities are more sensitive to the sampling and they can be attributed to statistical errors, which can be large along relatively short trajectories.

4.1.2. Structural Properties: Liquid Water. The determination of structural and dynamical properties of bulk water is a challenging task for computer simulations, due to the importance of many-body and cooperative effects that determine the peculiar characteristics of this liquid, such as high polarizability, self-dissociation, and hydrogen bonding patterns. The results obtained from $a b$ initio MD simulations crucially depend not only on thermodynamic conditions but also on several other factors such as the electronic structure method, ${ }^{22,44-47}$ the sampling method (e.g., Monte Carlo, CarParrinello MD, Born-Oppenheimer MD), ${ }^{46}$ the system size, ${ }^{48}$ and the total length of the trajectories. ${ }^{49}$ In particular, a number of DFT-based $a b$ initio MD studies ${ }^{22,4-46}$ have shown the large influence of the adopted XC functional on the predicted properties. At ambient conditions LDA gives largely overstructured radial distribution functions (RDFs) and very small self-diffusion constants, the footprint of a glassy-like behavior; GGA functionals perform slightly better, but, with some exceptions like the rPBE functional, ${ }^{44}$ provide RDFs that are still too structured compared to experimental data and diffusion coefficients that are roughly 10 times smaller than the 

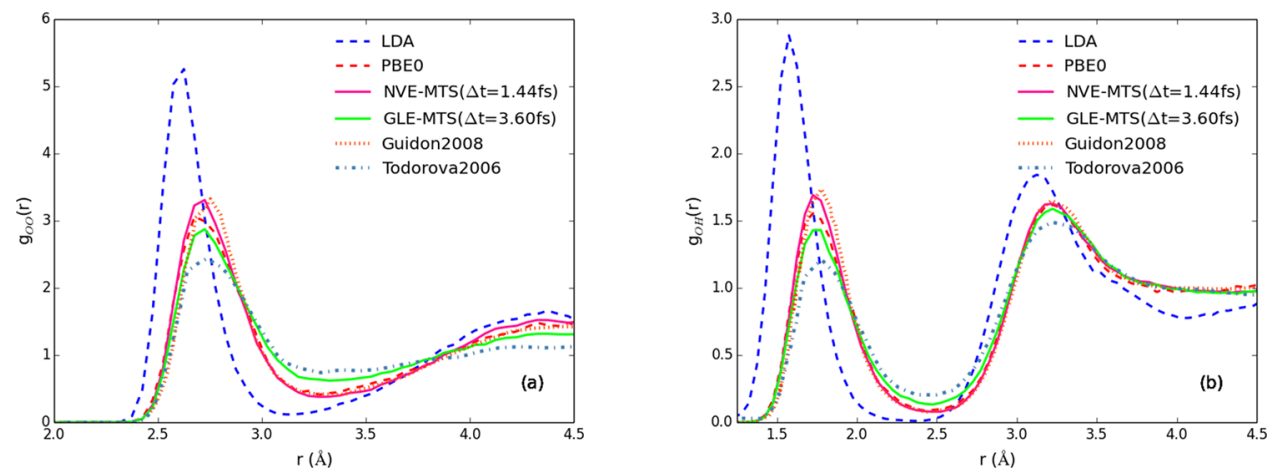

Figure 4. (a) Oxygen-oxygen and (b) intramolecular oxygen-hydrogen radial distribution functions obtained with LDA-VV MD (blue, dashed), PBE0-VV (red, dashed), LDA/PBE0 NVT-MTS MD (magenta), and GLE-MTS (green). For comparison, the same quantities from ref 22 (orange, dotted) and ref 44 (light blue, dot-dashed) are shown.

experimentally measured value, $D\left(T=45{ }^{\circ} \mathrm{C}\right)=0.2979 \AA^{2} /$ ps. $^{50}$ Hybrid functionals seem to be necessary to correctly reproduce structural and diffusive properties of liquid water, ${ }^{44}$ but there is no general consensus on their performances over a broad range of densities and temperatures. ${ }^{22}$ Weak interactions such as London dispersion, which are often not properly taken into account by standard exchange and correlation functionals, give also fundamental contributions to the properties of liquid water, and different routes to describe van der Waals interactions within DFT have been devised. ${ }^{51,52}$

Typically, within a plane wave formulation, the evaluation of exact exchange contributions to the XC functionals increases the CPU time by roughly a factor of 100 , compared to local or semilocal XC. Given the large differences between the LDAand hybrid-DFT description of water, we have chosen to apply the MTS strategy to liquid water considering LDA forces as a reference and PBE0 forces in the correction steps in order to assess the capabilities and limits of the MTS propagation scheme in the case of largely differing reference and correction systems.

Figure $4 \mathrm{a}$ and $\mathrm{b}$ show the oxygen-oxygen and the oxygenhydrogen $\mathrm{RDF}$ for a periodic system of 32 water molecules, as obtained from LDA, PBE0, LDA/PBE0-(NVE)MTS, and LDA/PBE0-(NVT)MTS (see Table 1 for trajectory details). For comparison, the RDFs of refs 22 and 44 are also shown.

Table 1. Average Temperature $\langle T\rangle$ in Kelvin and Length $t_{l}$ in ps of the Trajectory Used in Calculating the RDFs Shown in Figure 4

\begin{tabular}{lcr} 
& $\langle T\rangle(K)$ & $t_{l}(\mathrm{ps})$ \\
NVE-LDA & 366 & 20.0 \\
NVE-PBE0 & 357 & 8.0 \\
NVE-MTS & 352 & 20.0 \\
GLE-MTS & 372 & 20.0 \\
ref 44 & 350 & 5.0 \\
ref 22 & 325 & 7.5 \\
\hline
\end{tabular}

Compared with all the PBE0 curves, the LDA RDFs are much more structured as expected and the $\mathrm{OH} \mathrm{H}$-bonding distances as well as the OO distances in the first solvation shell are significantly shifted toward smaller values, as summarized in Tables 2 and 3. Maxima and minima of the RDFs of the MTS dynamics runs coincide with the analogous quantities in the reference PBE0 RDFs as well as with the results from refs 44 and 22. In particular, the overlap of the NVE-MTS curve with
Table 2. Position and Height of the First Maxima and Minima of the Oxygen-Oxygen RDFs Shown in Figure 4

\begin{tabular}{lcccc} 
& $r_{\max }$ & $g_{\mathrm{OO}}\left(r_{\max }\right)$ & $r_{\min }$ & $g_{\mathrm{OO}}\left(r_{\min }\right)$ \\
NVE-LDA & 2.63 & 5.26 & 3.13 & 0.12 \\
NVE-PBE0 & 2.68 & 3.07 & 3.27 & 0.41 \\
NVE-MTS & 2.73 & 3.39 & 3.28 & 0.35 \\
GLE-MTS & 2.73 & 2.87 & 3.33 & 0.62 \\
ref 44 & 2.74 & 2.58 & 3.35 & 0.73 \\
ref 22 & 2.75 & 3.32 & 3.39 & 0.42 \\
\hline
\end{tabular}

Table 3. Position and Height of the First Maxima and Minima of the Hydrogen-Oxygen RDFs Shown in Figure 4

\begin{tabular}{lcccc} 
& $r_{\max }$ & $g_{\mathrm{OH}}\left(r_{\max }\right)$ & $r_{\min }$ & $g_{\mathrm{OH}}\left(r_{\min }\right)$ \\
NVE-LDA & 1.58 & 2.88 & 2.38 & 0.01 \\
NVE-PBE0 & 1.73 & 1.56 & 2.43 & 0.09 \\
NVE-MTS & 1.73 & 1.69 & 2.53 & 0.08 \\
GLE-MTS & 1.78 & 1.43 & 2.48 & 0.13 \\
ref 44 & 1.78 & 1.21 & 2.37 & 0.20 \\
ref 22 & 1.78 & 1.73 & 2.43 & 0.08 \\
\hline
\end{tabular}

those of ref 22 is almost perfect. We can conclude that the MTS algorithm produces a reliable trajectory even in a limiting case when the reference forces would drive the dynamics toward a very different phase space region than the high level dynamics.

The combination of the MTS dynamics with a GLE colored noise thermostat ${ }^{26}$ allows stable dynamics to be maintained with an outer time step above the expected resonance threshold. A comparison of the RDFs from the GLE-MTS runs with the PBE0 reference shows that by using a GLE thermostat the structure of PBE0 liquid water is well reproduced also with an outer time step $\Delta t=3.6 \mathrm{fs}$, well above the resonance limit $(\Delta t \approx 2.7 \mathrm{fs})$. The less structured RDFs obtained from the GLE-MTS trajectory can be attributed to the slightly higher average temperature in this run compared to the reference ( $372 \mathrm{~K}$ vs $357 \mathrm{~K}$ ).

4.1.3. Water Dimer. The interface between CPMD and quantum chemistry codes such as G09 and TURBOMOLE developed here permits various combinations of density functional and $a b$ initio as well as semiempirical wave function based electronic structure methods. While CPMD uses a plane wave expansion of the Kohn-Sham orbitals and of the electronic densities, QC codes usually adopt atom-centered basis sets; i.e., through this interface also methods with different basis sets can be combined in an MTS scheme. Figure 5 compares the $\mathrm{OH}$ bond distributions along PBE/MP2-MTS1 

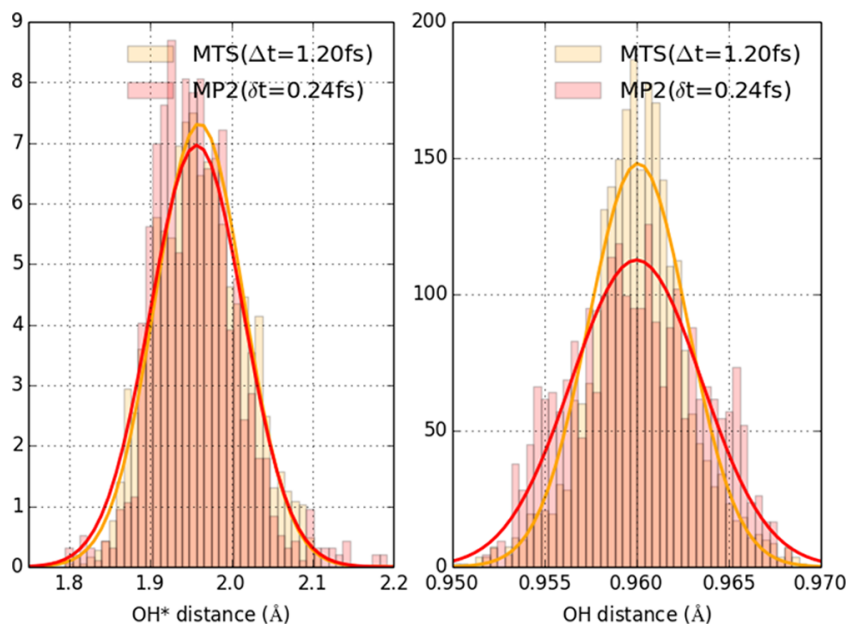

Figure 5. Comparison of the bond length distributions in a water dimer along PBE/MP2-MTS1 (equivalent to pure MP2) and PBE/ MP2-MTS5 trajectories of 20 ps.

and a MTS5 trajectories of the same length. In the figure we denote as MP2 the actual PBE/MP2-MTS1 results. MTS1 and VV propagations are indeed equivalent: when the correction is applied every step the contribution of the reference forces to the propagator cancels out and the dynamics is driven by the high level forces only. The average bond lengths extracted from the distributions, $d_{\mathrm{OH}^{*}}^{\mathrm{MP2}}=1.958(2) \AA$ and $d_{\mathrm{OH}^{*}}^{\mathrm{MTS}}=1.961(2) \AA$ for the hydrogen bond distance and $d_{\mathrm{OH}}^{\mathrm{MP} 2}=0.9617(1) \AA$ and $d_{\mathrm{OH}}^{\mathrm{MTS}}$ $=0.9617(1) \AA$ for the intramolecular OH bond, are compatible within the error bars, showing that also when both basis sets and electronic structure methods are different the MTS is able to provide the correct sampling.

4.2. Efficiency Evaluation. Defining $f=\tau_{\mathrm{vv}}^{L} / \tau_{\mathrm{vv}}^{H}$ as the ratio between the total simulation time in a MD run using the $\mathrm{VV}$ integrator and low level forces, $\tau_{\mathrm{vv}}^{L}$, and the total simulation time in a VV MD of same length with high level forces, $\tau_{\mathrm{vv}}^{H}$, the ideal speedup achieved by using the multiple time step approach can be calculated as

$$
s^{\mathrm{id}}=\frac{\tau_{\mathrm{vv}}^{H}}{\tau_{\mathrm{MTS}-\mathrm{n}}}=\frac{\tau_{\mathrm{vv}}^{H}}{\frac{1}{n} \tau_{\mathrm{vv}}^{H}+\tau_{\mathrm{vv}}^{L}}=\frac{n}{1+n f}
$$

If $f \rightarrow 0$, that is if the time spent in the calculation for the low level forces is negligible compared to the time needed for the high level ones, the ideal speedup tends to $n$. In practice however the maximum value of $n$ that can be used is limited by resonances that are occurring when the microcanonical rRESPA scheme is adopted and that can be overcome, as demonstrated above, by the use of colored noise thermostats. ${ }^{25,26}$ However, the main limitation to the speedup in the present MTS implementation is related to the number of self-consistency (SC) cycles needed to reconverge the wave function after a nuclear displacement. For larger steps, the number of SC cycles becomes larger, as an initial guess of the wave function based on its history recorded in one or more previous steps becomes less accurate.

Figure 6a compares the ideal and the real speedup for the test systems presented here (acetone in vacuum and in aqueous solution). The factor of roughly 2 that separates the real speedup from the ideal one is due to the essentially linear increase of the number of SC iterations as a function of $\Delta t$ : this is an intrinsic problem, also present in the Velocity Verlet propagation. We explored several possibilities trying to maximize MTS efficiency: Figure $6 \mathrm{~b}$ shows the LDA/PBE0MTS speedup for a system of 32 water molecules. The various curves for the real speedup illustrate different ways to obtain the initial guess of the high level wave function: standard extrapolation based on the history of the high level wave function (red squares), initial guess from the low level wave function (dotted red line), same extrapolation as for the low level wave function (dashed blue line), and mixing schemes (dash-dotted red line). The nonstandard schemes improve the speedup at large time steps, raising the MTS efficiency to $70 \%$.

\section{CONCLUSIONS}

We presented an implementation of a multiple time step scheme for $a b$ initio molecular dynamics simulations in both a full $\mathrm{QM}$ and a $\mathrm{QM} / \mathrm{MM}$ context, based on the time-reversible RESPA algorithm originally developed for classical MD. In the presented scheme, nuclear forces are evaluated $a b$ initio, at different levels of theory, both in the reference and in the correction steps. Being fully based on $a b$ initio forces, this MTS scheme can be applied for a wide range of situations including chemical reactions.

The algorithm has been implemented in a development version of the latest release (version 4.1) of the DFT-based code for $a b$ initio molecular dynamics CPMD and allows for a 5- to 6-fold speedup in realistic (bulk) systems where the evaluation of the high level forces in the correction step is the dominant contribution to the total computational time. This speedup corresponds to a significant saving of CPU and human time, with no impact on the sampling accuracy. A newly
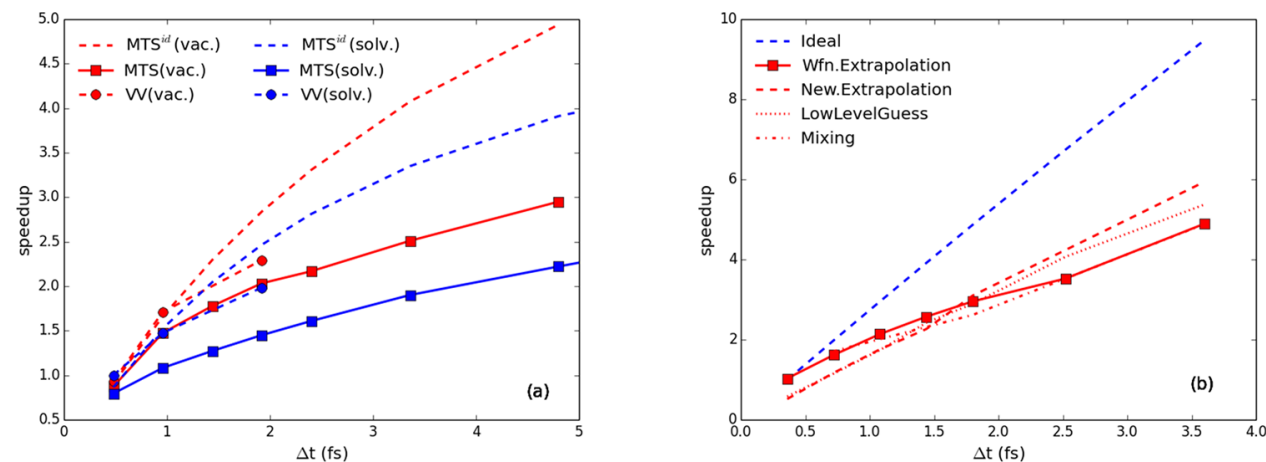

Figure 6. MTS speedup. (a) Acetone molecule in vacuum (red curves) and in water (blue curves); (b) 32 water molecules, relative to different initial guesses of the wave function. 
developed interface with quantum chemistry codes (G09, TURBOMOLE, ...) also makes it possibile to go beyond DFT accuracy at a strongly reduced computational cost.

We found two major limitations to the maximum speedup of the method. The first, intrinsic to the MTS procedure and already well described in the literature, is due to resonances of residual high frequency force components with the outer time step. Several schemes have been devised to overcome resonance problems in classical MTS MD, such as the use of stochastic thermostats. ${ }^{25,26}$ We showed that applying a GLE colored noise thermostat ${ }^{26}$ in combination with this MTS scheme leads to a correct reproduction of the structure of PBE0 liquid water even when using an outer time step $\Delta t=3.6 \mathrm{fs}$, well above the expected resonance threshold in water $\left(\Delta t_{\max } \approx\right.$ 2.7 fs). Combination with the Stochastic-Iso-NH-RESPA thermostat ${ }^{25}$ could also be a promising approach to further enlarge the outer time step and reach even larger speedups.

The second limitation to the maximum speedup is due to the initial guess of the (high level) wave function, which becomes less accurate as the outer time step increases. This issue is not present when the interface with QC codes is used to evaluate the forces in the correction steps, but only when they are computed within the CPMD code and it has already been pointed out in other AIMD-MTS implementations. ${ }^{53}$ We explored several alternative approaches that might help improve the situation for very large outer time steps. The development of more powerful wave function extrapolation schemes, beyond the scope of the present paper, can further improve the method efficiency bringing the speedup closer to its ideal limit. However, even under nonoptimal conditions we proved that the present MTS scheme is a versatile and powerful tool, able to speed up $a b$ initio $\mathrm{MD}$ simulations by almost an order of magnitude, without impacting the computed properties.

\section{ASSOCIATED CONTENT}

\section{(S) Supporting Information}

The Supporting Information is available free of charge on the ACS Publications website at DOI: 10.1021/acs.jctc.7b01189.

Test for a different placement of the thermostat operator in the Trotter factorization, known as middle-scheme, and an alternative decomposition of the total force (these modifications do not improve significantly the scheme presented in the main text) (PDF)

\section{AUTHOR INFORMATION}

\section{Corresponding Author}

*E-mail: ursula.roethlisberger@epfl.ch.

ORCID 1

Rocco Meli: 0000-0002-2845-3410

Ursula Rothlisberger: 0000-0002-1704-8591

Notes

The authors declare no competing financial interest.

\section{ACKNOWLEDGMENTS}

The authors thank Prof. M. Ceriotti for useful discussions concerning the GLE thermostat. E.L acknowledges Dr. P. Lopez-Tarifa for his help in the early stages of MTS testing. U.R. acknowledges financial support from the Swiss National Science foundation Grant No. 200020-146645 and the NCCR MUST. Computer resources were provided by the DIT/EPFL, the CSCS, and the CADMOS project.

\section{REFERENCES}

(1) Swope, W. C.; Andersen, H. C.; Berens, P. H.; Wilson, K. R. A computer simulation method for the calculation of equilibrium constants for the formation of physical clusters of molecules: Application to small water clusters. J. Chem. Phys. 1982, 76, 637-649.

(2) Schlick, T.; Mandziuk, M.; Skeel, R. D.; Srinivas, K. Nonlinear Resonance Artifacts in Molecular Dynamics Simulations. J. Comput. Phys. 1998, 140, 1-29.

(3) Ryckaert, J.-P.; Ciccotti, G.; Berendsen, H. J. C. Numerical Integration of the Cartesian Equations of Motion of a System with Constraints: Molecular Dynamics of n-Alkanes. J. Comput. Phys. 1977, 23, 327-341.

(4) Andersen, H. C. RATTLE: A Velocity Version of the SHAKE Algorithm for Molecular Dynamics Calculations. J. Comput. Phys. 1983, 52, 24-34.

(5) Van Gusteren, W. F.; Karplus, M. Effect of Constraints on the Dynamics of Macromolecules. Macromolecules 1982, 15, 1528-1544.

(6) Toxvaerd, S. Comment on constrained molecular dynamics of macromolecules. J. Chem. Phys. 1987, 87, 6140-6143.

(7) Tuckerman, M. E.; Martyna, G. J.; Berne, B. J. Molecular dynamics algorithm for condensed systems with multiple time scales. J. Chem. Phys. 1990, 93, 1287-1291.

(8) Tuckerman, M. E.; Berne, B. J.; Rossi, A. Molecular dynamics algorithm for multiple time scales: Systems with disparate masses. J. Chem. Phys. 1991, 94, 1465-1469.

(9) Tuckerman, M. E.; Berne, B. J.; Martyna, G. J. Molecular dynamics algorithm for multiple time scales: Systems with long range forces. J. Chem. Phys. 1991, 94, 6811-6815.

(10) Tuckerman, M. E.; Berne, B. J. Molecular dynamics in systems with multiple time scales: Systems with stiff and soft degrees of freedom and with short and long range forces. J. Chem. Phys. 1991, 95, 8362-8364.

(11) Tuckerman, M. E.; Berne, B. J.; Martyna, G. J. Reversible multiple time scale molecular dynamics. J. Chem. Phys. 1992, 97, 1990-2001.

(12) Streett, W.; Tildesley, D.; Saville, G. Multiple time-step methods in molecular dynamics. Mol. Phys. 1978, 35, 639-648.

(13) Grubmüller, H.; Heller, H.; Windemuth, A.; Schulten, K. Generalized Verlet Algorithm for Efficient Molecular Dynamics Simulations with Long-range Interactions. Mol. Simul. 1991, 6, 121142.

(14) Humphreys, D. D.; Friesner, R. A.; Berne, B. J. A MultipleTime-Step Molecular Dynamics Algorithm for Macromolecules. J. Phys. Chem. 1994, 98, 6885-6892.

(15) García-Archilla, B.; Sanz-Serna, J. M.; Skeel, R. D. Long-timestep methods for oscillatory differential equations. SIAM J. Sci. Comput. 1998, 20, 930-963.

(16) Izaguirre, J. A.; Reich, J.; Skeel, R. D. Longer time steps for molecular dynamics. J. Chem. Phys. 1999, 110, 9853-9864.

(17) Tuckerman, M.; Parrinello, M. Integrating the Car-Parrinello equations. II. Multiple time scale techniques. J. Chem. Phys. 1994, 101, 1316.

(18) Luehr, N.; Markland, T. E.; Martínez, T. J. Multiple time step integrators in ab initio molecular dynamics. J. Chem. Phys. 2014, 140, 084116.

(19) Steele, R. P. Communication: Multiple-timestep ab initio molecular dynamics with electron correlation. J. Chem. Phys. 2013, $139,011102$.

(20) Steele, R. P. Multiple-Timestep ab Initio Molecular Dynamics Using an Atomic Basis Set Partitioning. J. Phys. Chem. A 2015, 119, 12119-12130.

(21) Fatehi, S.; Steele, R. P. Multiple-Time Step Ab Initio Molecular Dynamics Based on Two-Electron Integral Screening. J. Chem. Theory Comput. 2015, 11, 884-898.

(22) Guidon, M.; Schiffmann, F.; Hutter, J.; VandeVondele, J. Ab initio molecular dynamics using hybrid density functionals. J. Chem. Phys. 2008, 128, 214104.

(23) Moeller, C.; Plesset, M. S. Note on an Approximation Treatment for Many-Electron Systems. Phys. Rev. 1934, 46, 618-622. 
(24) Minary, P.; Tuckerman, M. E.; Martyna, G. J. Long Time Molecular Dynamics for Enhanced Conformational Sampling in Biomolecular Systems. Phys. Rev. Lett. 2004, 93, 150201.

(25) Leimkuhler, B.; Margul, D. T.; Tuckerman, M. E. Stochastic, resonance-free multiple time-step algorithm for molecular dynamics with very large time steps. Mol. Phys. 2013, 111, 3579-3594.

(26) Morrone, J. A.; Markland, T. E.; Ceriotti, M.; Berne, B. J. Efficient multiple time scale molecular dynamics: Using colored noise thermostats to stabilize resonances. J. Chem. Phys. 2011, 134, 014103.

(27) CPMD V 4.1, http://www.cpmd.org/. Copyright IBM Corp. 1990-2015, Copyright MPI für Festkürperforschung Stuttgart 19972001.

(28) Kohn, W.; Sham, L. J. Self-consistent equations including exchange correlation effects. Phys. Rev. 1965, 140, A1133-1138.

(29) Ceriotti, M.; Bussi, G.; Parrinello, M. Langevin equation with colored noise for constant-temperature molecular dynamics simulations. Phys. Rev. Lett. 2009, 102, 020601.

(30) Ceriotti, M.; Bussi, G.; Parrinello, M. Colored-noise thermostats à la carte. J. Chem. Theory Comput. 2010, 6, 1170-1180.

(31) Perdew, J. P.; Ernzerhof, M.; Burke, K. Rationale for mixing exact exchange with density functional approximations. J. Chem. Phys. 1996, 105, 9982-9985.

(32) Perdew, J. P.; Burke, K.; Ernzerhof, M. Generalized gradient approximation made simple. Phys. Rev. Lett. 1996, 77, 3865-3868.

(33) Frisch, M. J.; Trucks, G. W.; Schlegel, H. B.; Scuseria, G. E.; Robb, M. A.; Cheeseman, J. R.; Scalmani, G.; Barone, V.; Petersson, G. A.; Nakatsuji, H.; Li, X.; Caricato, M.; Marenich, A.; Bloino, J.; Janesko, B. G.; Gomperts, R.; Mennucci, B.; Hratchian, H. P.; Ortiz, J. V.; Izmaylov, A. F.; Sonnenberg, J. L.; Williams-Young, D., Ding, F.; Lipparini, F.; Egidi, F.; Goings, J.; Peng, B.; Petrone, A.; Henderson, T.; Ranasinghe, D.; Zakrzewski, V. G.; Gao, J.; Rega, N.; Zheng, G.; Liang, W.; Hada, M.; Ehara, M.; Toyota, K.; Fukuda, R.; Hasegawa, J.; Ishida, M.; Nakajima, T.; Honda, Y.; Kitao, O.; Nakai, H.; Vreven, T.; Throssell, K.; Montgomery, J. A., Jr.; Peralta, J. E.; Ogliaro, F.; Bearpark, M.; Heyd, J. J.; Brothers, E.; Kudin, K. N.; Staroverov, V. N.; Keith, T.; Kobayashi, R.; Normand, J.; Raghavachari, K.; Rendell, A.; Burant, J. C.; Iyengar, S. S.; Tomasi, J.; Cossi, M.; Millam, J. M.; Klene, M.; Adamo, C.; Cammi, R.; Ochterski, J. W.; Martin, R. L.; Morokuma, K.; Farkas, O.; Foresman, J. B.; Fox, D. J. Gaussian 09, revision B02; Gaussian, Inc.: Wallingford, CT, 2016.

(34) TURBOMOLE V6.2 2010, a development of University of Karlsruhe and Forschungszentrum Karlsruhe GmbH, 1989-2007, TURBOMOLE GmbH, since 2007; available from http://www. turbomole.com.

(35) Nosé, S. A unified formulation of the constant temperature molecular dynamics methods. J. Chem. Phys. 1984, 81, 511.

(36) Nosé, S. A molecular dynamics method for simulations in the canonical ensemble. Mol. Phys. 1984, 52, 255.

(37) Hoover, W. G. Canonical dynamics: Equilibrium phase-space distributions. Phys. Rev. A: At., Mol., Opt. Phys. 1985, 31, 1695.

(38) Martyna, G. J.; Tuckerman, M. E.; Tobias, D. J.; Klein, M. L. Explicit reversible integrators for extended systems dynamics. Mol. Phys. 1996, 87, 1117-1157.

(39) Zhang, Z.; Liu, X.; Chen, Z.; Zheng, H.; Yan, K.; Liu, J. A unified thermostat scheme for efficient configurational sampling for classical/ quantum canonical ensembles via molecular dynamics. J. Chem. Phys. 2017, 147, 034109.

(40) Ma, Q.; Izaguirre, J. A.; Skeel, R. D. Verlet-I/R-RESPA/Impulse is Limited by Nonlinear Instabilities. SIAM J. Sci. Comput. 2003, 24, 1951-1973.

(41) Troullier, N.; Martins, J. L. Efficient pseudopotentials for plane wave calculations. Phys. Rev. B: Condens. Matter Mater. Phys. 1991, 43, 1993-2006.

(42) Martyna, G.; Tuckerman, M. E. A reciprocal space based method for treating long range interactions in $a b$ initio and force-fieldbased calculations in clusters. J. Chem. Phys. 1999, 110, 2810.

(43) Jorgensen, W. L.; Chandrasekhar, J.; Madura, J. D. Comparison of simple potential functions for simulating liquid water. J. Chem. Phys. 1983, 79, 926 .
(44) Todorova, T.; Seitsonen, A. P.; Hutter, J.; Kuo, I.-F. W.; Mundy, C. J. Molecular Dynamics Simulation of Liquid Water: Hybrid Density Functionals. J. Phys. Chem. B 2006, 110, 3685-3692.

(45) Sprik, M.; Hutter, J.; Parrinello, M. Ab initio molecular dynamics simulation of liquid water: Comparison of three gradientcorrected density functionals. J. Chem. Phys. 1996, 105, 1142.

(46) Kuo, I.-F. W.; Mundy, C. J.; McGrath, M. J.; Siepmann, J. I.; VandeVondele, J.; Sprik, M.; Hutter, J.; Chen, B.; Klein, M. L.; Mohamed, F.; Krack, M.; Parrinello, M. J. Phys. Chem. B 2004, 108, 12990-12998.

(47) Del Ben, M.; Schönherr, M.; Hutter, J.; VandeVondele, J. Bulk Liquid water at Ambient Temperature and Pressure from MP2 Theory. J. Phys. Chem. Lett. 2013, 4, 3753.

(48) Silvestrelli, P. L.; Parrinello, M. Structural, electronic, and bonding properties of liquid water from first principles. J. Chem. Phys. 1999, 111, 3572.

(49) Schwegler, E.; Grossman, J. C.; Gygi, F.; Galli, G. Towards an assessment of the accuracy of density functional theory for first principles simulations of water. II. J. Chem. Phys. 2004, 121, 5400.

(50) Stillinger, F. H. Water Revisited. Science 1980, 209, 451-457.

(51) Zhang, C.; Wu, J.; Galli, G.; Gygi, F. Structural and Vibrational Properties of Liquid Water from van der Waals Density Functionals. $J$. Chem. Theory Comput. 2011, 7, 3054-3061.

(52) Lin, I.-C.; Seitsonen, A. P.; Tavernelli, I.; Rothlisberger, U. Structure and dynamics of liquid water from ab Initio molecular dynamics comparison of BLYP, PBE, and revPBE Density Functionals with and without van der Waals corrections. J. Chem. Theory Comput. 2012, 8, 3902-3910.

(53) Nam, K. Acceleration of Ab Initio QM/MM Calculations under Periodic Boundary Conditions by Multiscale and Multiple Time Step Approaches. J. Chem. Theory Comput. 2014, 10, 4175-4183. 